\title{
Tobacco use and COVID-19 in Ghana: generating evidence to support policy and practice
}

\author{
Arti Singh ${ }^{1}$ (1) Divine Darlington Logo ${ }^{2}$, Fiona Dobbie ${ }^{3}$, Rob Ralston ${ }^{3}$, Fiona Davidson ${ }^{3}$, Linda Bauld ${ }^{3}$, Ellis Owusu-Dabo ${ }^{1}$ \\ 1 School of Public Health, KNUST, Ghana, ${ }^{2}$ Research and Development Division, Ghana Health Service, Accra, Ghana, ${ }^{3}$ Usher Institute and SPECTRUM \\ Consortium, University of Edinburgh, Edinburgh, UK \\ Keywords: covid-19, tobacco, policy, ghana \\ https://doi.org/10.29392/001c.19141
}

\section{Journal of Global Health Reports}

Vol. 5, 2021

\section{Background}

The COVID-19 pandemic has affected over 45 million people and caused over a million deaths globally. Tobacco use, a threat to public health worldwide, increases the risk of developing severe COVID-19 disease and death. The hand-to-mouth action, smoking-induced lung diseases, and the sharing of tobacco products such as water pipes, increase a smoker's vulnerability to COVID-19. Implementation of tobacco control efforts in low- and middle-income countries (LMICs) including sub-Saharan Africa (SSA) is a particular challenge. The aim of this study in Ghana was to produce evidence to support governments to make informed policy decisions about tobacco control interventions in the context of COVID-19.

\section{Methods}

A survey with key stakeholders (conducted online or via face to face interview) and a desk-based mapping of data sources including government reports and online print press. Face-to Face interviews followed the COVID-19 precautionary protocols.

\section{Results}

40 stakeholders participated in the interviews ( 28 online and 12 face-to-face). 46 data sources were identified from the mapping of which 16 were eligible for data extraction. Over two fifths of survey respondents $(42.9 \%, n=12)$ agreed that the relationship between COVID-19 and tobacco use had been discussed in the media, and over half $(57 \%, n=16)$ reported that public health professionals and other authorities had provided advice to tobacco users during the pandemic. While respondents $(89 \%, \mathrm{n}=25)$ did not see a change in the level of interest in tobacco cessation, less than a quarter $(23 \%, n=6)$ indicated that the policy response to COVID-19 included a focus on tobacco control issues, but was limited to tobacco cessation. The majority of respondents $(77 \%, n=31)$ reported a perceived limited impact on the tobacco industry's operations during the pandemic.

\section{Conclusions}

COVID-19 provides a timely opportunity to strengthen tobacco control efforts by recognizing the role of tobacco use in potentially exacerbating covid-19 health outcomes and promoting cessation.

The coronavirus (COVID-19) pandemic that began in December 2019 presents an immediate risk to global health with far-reaching, and long-term, health and economic consequences. Since $31^{\text {st }}$ December 2019 and as of October 2020 , over 42 million cases of COVID-19 have been reported worldwide, with over a million deaths. ${ }^{1}$ Africa was the last continent to be affected by the virus and as of $30^{\text {th }}$ October 2020 had over a 1.5 million cases and over 40,000 deaths, with a $2 \cdot 4 \%$ case fatality, representing $5 \%$ of global infections. $^{2}$

The pandemic has affected almost all aspects of life for much of the world's population. Although evidence on who is most vulnerable to COVID-19 is still emerging, the presence of underlying health conditions such as diabetes, heart or respiratory infections and cancers, puts individuals at more risk. ${ }^{3}$ Tobacco use, is a major risk factor for all the listed chronic conditions. ${ }^{4}$ A recent rapid evidence review, of 345 studies, conducted by University College London (UCL), noted uncertainty about the associations of smoking status with COVID-19 outcomes, particularly in relation to the potential therapeutic effect of nicotine. ${ }^{5}$ They cautioned that further research is needed and noted that it was 'premature' to speculate about the potential protective effects from nicotine, due to the quality of the existing evi- 
dence. However, their review and others indicates that active smoking and a history of smoking were associated with worse outcomes from COVID-19. Thus, the SARS-COV-2 epidemic should serve as an impetus for patients and those at risk to maintain good health practices and discontinue smoking. ${ }^{6}$ The World Health Organization (WHO) has further highlighted that the hand-to-mouth action, smokinginduced lung disease, and the sharing of tobacco products such as water pipes, as factors which may increase a smoker's vulnerability to SARS-CoV-2 infection and development of COVID-19. ${ }^{7}$

The sub-Saharan African (SSA) region, despite being in the early stage of the tobacco epidemic, is particularly vulnerable due to the its weak tobacco laws, and is expected to face the largest growth of tobacco consumption in the world. ${ }^{8}$ Ghana, a low and middle-income country in West Africa, has less than $10 \%$ of its population smoking ${ }^{9}$ and more than 5,000 people dying from tobacco-related diseases every year. ${ }^{10}$ Although, cigarettes remain the commonest form of tobacco use, other forms of tobacco products such as water pipe smoking (shisha) are becoming common particularly amongst the youth. ${ }^{11}$ The regional trend in the country continues to show high prevalence from the northern part of the country - 31.2\%, $22.5 \%$ and 7.9\% in the Upper East, Northern and Upper West regions respectively as compared to the other regions in Ghana. ${ }^{12}$ In the face of the current pandemic (COVID-19), most resource-limited nations in SSA including Ghana may be unable to prioritize adequate resources to address tobacco control. Considering the wider implications of tobacco control on population health and the healthcare system, it is crucial to optimize policies influencing tobacco use during the pandemic. With Ghana's COVID-19 cases still increasing, there is a need to better integrate responses to such pandemics and tobacco control interventions, particularly in SSA where health capacity is limited and smoking prevalence is rising.

This study builds on an existing GCRF-funded Tobacco Control Capacity Programme (TCCP), which is designed to support research institutions in LMICs to develop policyrelevant research in tobacco control. ${ }^{13}$ The TCCP is a consortium of 15 partners, nine of which are in Africa and Asia. The current study aims to generate evidence to support governments in LMICs to make informed policy decisions about the tobacco control interventions, in the context of COVID-19 or other future infectious disease epidemics that affect respiratory health. The specific objectives of the project are to

- Examine the relationship between COVID-19 and tobacco use in Ghana

- Explore how efforts to address COVID-19 or other future infectious disease epidemics can be integrated with tobacco control policies and interventions in Ghana.

Findings and recommendations from the study will be of interest to policy makers, professionals and civil society groups in Ghana and beyond, who are concerned about the links between COVID-19 and tobacco use and progressing tobacco control while also responding to the pandemic.

\section{METHODS}

This was a mixed method study comprised of two elements: stakeholder consultation via an online survey, face to face (where applicable) or telephone interviews; and desk-based mapping to identify any relevant unpublished data sources such as government reports and online print press. The survey covered issues to do with the relationship between COVID-19 and tobacco use, impact of the COVID-19 pandemic on the policy response to tobacco control and implications of the COVID-19 pandemic on development of tobacco control policy. A descriptive analysis of survey findings was undertaken. The qualitative data (from face-toface interviews) were analysed using a systematic step-bystep review of the data and identification of themes related to the study topic and presented as proportions. The desk based mapping of unpublished data sources (such as government reports, online print press and twitter) on COVID-19 and tobacco was guided by a protocol and conducted in three rounds between May and July 2020. The study was approved by the Ethics committee of the Kwame Nkrumah University of Science and Technology in Ghana. Written and verbal informed consent was sought from each respondents interviewed for the study. Box 1 summaries the mapping approach. 
Table 1. Stakeholder survey response sample characteristics

\begin{tabular}{|c|c|c|c|}
\hline Response & Area & Institutional affiliation & Government level \\
\hline $\begin{array}{l}50 \text { invitations sent } \\
40 \text { stakeholders took } \\
\text { part } \\
\text { - } 28 \text { online survey } \\
\text { - } 12 \text { interview }\end{array}$ & $\begin{array}{l}\text { Accra }(42.5 \%, n=17) \\
\text { Other regions }(20 \% \\
n=8) \\
\text { Missing }(7.5 \%, n=3)\end{array}$ & $\begin{array}{l}\text { Civil society }(12.5 \%, n=5) \\
\text { Government }(45 \%, n=18) \\
\text { Health professional }(32.5 \% \text {, } \\
n=13) \\
\text { Other }(10 \%, n=4)\end{array}$ & $\begin{array}{l}\text { National }(57.5 \%, n=23) \\
\text { Regional / District } 12.5 \% \text {, } \\
n=5)\end{array}$ \\
\hline
\end{tabular}

\section{Box 1. Summary of approach and data sources in Ghana}

Three rounds of data search were conducted. The first, conducted on $15^{\text {th }}$ May 2020, searched for data sources published between 31 January 2020 (chosen because this was the date that the WHO declared COVID-19 to be a public health emergency of international concern) and 01 June 2020. The second search was conducted on 22 June 2020, and the final search was conducted on 01 July 2020.

\section{Sources of information}

- Google search using the 'All' and 'News' tab

- Website search: Ghanaian Ministry of Information website, the Ghanaian Ministry of Health website, the WHO Country Office for Ghana website, WHO FCTC and NGO websites: Centre for Tobacco Control Africa, AMREF Health Africa, African Tobacco Control Alliance, Tobacco Atlas and Ghana Non-Communicable Disease Alliance.

- Online print press using Google alerts

- Twitter accounts for Ghanaian Ministry of Health, Ghanaian Ministry of Information, WHO Ghana, and the Ghana NCD Alliance

\section{- Consultation with colleagues in Ghana}

Number of data sources identified: 46

Number eligible for data extraction: 16 (mainly online print press and twitter)

\section{RESULTS}

A total 50 stakeholders were contacted of which 40 agreed to participate in the survey. Of the 40 interviews, 28 were conducted online and 12 were face-to-face. Table 1 provides the characteristics of the study participants.

\section{WHAT DO WE KNOW ABOUT COVID-19 AND TOBACCO USE IN GHANA?}

The review did not identify any publications that were specific to COVID-19 and tobacco in Ghana.

\section{IMPACT OF COVID-19 ON TOBACCO CONTROL POLICY}

Over two fifths of survey respondents in Ghana (42.9\%, $\mathrm{n}=12$ ) agreed that the relationship between COVID-19 and tobacco use had been discussed in the media, and over half $(57 \%, n=16)$ reported that public health professionals and other authorities had provided advice to tobacco users during the pandemic. Participants who took part in an interview also agreed and noted that health professionals had been taking part in media discussions, which concurs with the mapping that identified Twitter in Ghana as an important medium for sharing information and advice. Our study found no evidence of a population level policy response to date. This finding is not surprising, considering that just under one quarter of survey respondents $(23 \%$, $n=6)$ thought that the policy response to COVID-19 included a focus on tobacco control issues, but was limited to tobacco cessation. Stakeholders also recommended increments in tobacco taxes (ideally by $50 \%$ ) to generate revenue to support healthcare delivery in the wake of Covid-19.

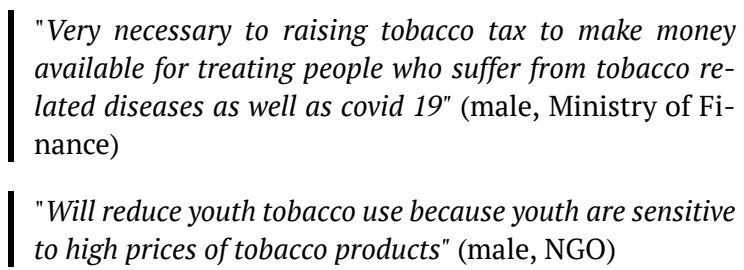

Another key suggestion from stakeholders was to review the current pictorial health warning on cigarette packs to standard plain packaging as recommended by the WHO Framework Convention on Tobacco Control. This will be important for youth tobacco use prevention, as other countries that have implemented standardised packaging have noted.

\section{ADVICE ON TOBACCO CESSATION}

The key piece of advice given by the stakeholders, in the face of the COVID-19 pandemic being more severe on smokers, was to stop using tobacco products. However, survey findings suggest that respondents did not see a change in the level of interest in tobacco cessation with $89 \%(n=25)$ reporting no change or saying that they did not know. Similar perspectives came from interview participants. However, interviewees noted that lockdown restrictions offered an opportunity to promote cessation, in the context of reduced tobacco sales.

"Pubs, drinking bars and restaurants and hotels closed, hence reduced sales of tobacco leading to reduced smok- 
Ing” (male, Disease Control office, Ghana Health Service)

The mapping element of the research identified calls from the Ghana NCD Alliance and the Vision for Alternative Development (VALD) for the government to strengthen or enforce existing tobacco control measures. These included: a ban on tobacco use (including shisha); measures to protect children from the use of and exposure to cigarettes; enforcement of the ban on the sale of single sticks of cigarette products and ban the sale of 10 sticks of cigarettes; protection of public health policies from tobacco industry interference; strengthen collaboration between government and civil society organisations to improve surveillance; reporting and enforcement mechanisms.

\section{TOBACCO INDUSTRY RESPONSE TO COVID-19}

Survey respondents reported limited impact on the way the tobacco industry operated in Ghana during the COVID-19 crisis, with three quarters $(77 \%, \mathrm{n}=31)$ saying it had no effect or they did not know. This concurs with the mapping that did not find any data sources reporting on tobacco industry response to COVID-19. However, interview participants were more likely to report that tobacco sales may have been affected as a result of public health responses to COVID-19 including the closure of all bars, pubs and other hospitality venues. In addition, disruption of international trade and border controls may have temporarily affected tobacco product imports. As one interviewee stated:

"Closure of borders and public gatherings may reduce tobacco sale. People are aware that tobacco can easily lead to COVID-19 infection hence many users try to avoid tobacco". (Female/Food and Drugs Authority)

\section{CONCLUSIONS}

While tobacco use in Ghana is lower than in many other countries, ${ }^{8}$ it is imperative that national governments do not neglect their responsibility to prevent premature deaths from tobacco, and the morbidity associated with tobacco use. This is particularly important at the current time when tobacco use can, from existing evidence, affect COVID-19 disease severity. ${ }^{3}$ Although, COVID-19 provides opportunity to promote tobacco cessation in Ghana as indicated by the stakeholders, this may be a challenge given the pandemic-associated stress, physical isolation, uncertainty about the future, and rising unemployment, which is associated with increased smoking prevalence. ${ }^{14}$ The COVID-19 pandemic highlights the importance of health behaviors and creates an added sense of urgency to strengthen public health policies (at organizational, healthcare team, and individual levels).

\section{Box 2. Recommendations}

- Smoking cessation support programs need to be heightened in the face of the pandemic. For example, opportunity for smoking cessation support should be integrated into all health care settings and through the use of diverse health professionals (such as public health, medicine and psychology) and targeted media channels. For example, tailored messaging and awareness building programs about the relationship between smoking and COVID-19 and integrating messages about the importance of smoking cessation and supports available into COVID-19 testing and/or contact tracing services. Again, given the important role played by the media and formal education in discouraging smoking behavior, these media could be used more intensively in disseminating relevant information on the dangers of smoking.

- Consider the potential impact of a temporary ban on sales and importation of tobacco products including cigarettes during the COVID-19 crisis, noting that, one the one hand, COVID-19 is a respiratory illness and tobacco could aggravate symptoms and worsen outcomes, but also that abrupt nicotine withdrawal could lead also lead to poorer outcomes. ${ }^{15}$ COVID-19 presents an opportunity to ban products such as shisha as it use prevails among youth, ${ }^{16}$ and involves sharing of the same mouthpiece, which can be a route for SARS-CoV-2 transmission and other communicable diseases.

- The tobacco industry has an irreconcilable conflict of interest with public health and has a well-documented history of deception and of capitalizing on humanitarian crises, natural disasters and other similar catastrophic events. ${ }^{17}$ Policy makers and tobacco control advocates in Ghana need to protect tobacco control efforts from the commercial and other vested interests of the tobacco industry.

- Future studies in Ghana and beyond are needed to better understand the relationship between tobacco-related diseases and COVID-19, including data that can compare survival outcomes between COVID-19 positives cases who are smokers and those who are non-smokers. More detail on the smoking status of COVID-19 cases (i.e. how many were current smokers or smokers in the past) is required to contribute to emerging international debates about the implications of COVID-19 for tobacco control and non-communicable disease agendas. 


\section{ACKNOWLEDGMENTS}

The authors thank all the stakeholders for providing verbal information on tobacco control in Ghana.

\section{FUNDING}

This work was supported by the Medical Research Council [grant number MR/P027946/2] with funding from the Global Challenges Research Fund and with additional funding from the University of Edinburgh's Scottish Funding Council Global Challenges Research Fund (GCRF) allocation.

\section{AUTHORSHIP CONTRIBUTIONS}

AS drafted the initial version of the manuscript. FD, DDL and LB contributed to the revision of the manuscript for important intellectual content and final approval. All other authors reviewed the final draft for approval.

\section{COMPETING INTERESTS}

The authors completed the Unified Competing Interest form at www.icmje.org/coi_disclosure.pdf (available upon request from the corresponding author), and declare no conflicts of interest.

\section{CORRESPONDENCE TO}

Dr Arti Singh

School of Public Health

KNUST, Ghana

Email: artisingh uk@yahoo.com

Submitted: November 28, 2020 GMT, Accepted: February 02, 2021 GMT 


\section{REFERENCES}

1. World Health Organization. WHO Coronavirus Disease (COVID-19) Dashboard. World Health Organization; 2020.

2. WHO. COVID-19 COVID-19. WHO journal for covid 19, Region Situational update for the Africa. Published online 2020.

3. Vardavas CI, Nikitara K. COVID-19 and smoking: A systematic review of the evidence. Tobacco Induced Diseases. Published online 2020. doi:10.18332/tid/119 $\underline{324}$

4. West R, Hardy A. Tobacco use. In: Cambridge Handbook of Psychology, Health and Medicine. Second Edition. ; 2014. https://doi:10.1017/CBO97805115435 $\underline{79.244}$

5. Simons D, Lion S, Jamie B, Olga P. The association of smoking status with SARS-CoV-2 infection, hospitalisation and mortality from COVID-19: A living rapid evidence review with Bayesian metaanalyses (version 10). Qeios.

6. Patanavanich R, Glantz SA. Smoking Is Associated With COVID-19 Progression: A Meta-analysis. Nicotine \& tobacco research: official journal of the Society for Research on Nicotine and Tobacco. Published online 2020. doi:10.1093/ntr/ntaa082

7. WHO. Smoking and COVID-19:scientific brief. World Health Organisation. Published online 2020.

8. Singh A, Owusu-Dabo E, Dobbie F, et al. A situational analysis of tobacco control in Ghana: progress, opportunities and challenges. Journal of Global Health Reports. Published online 2020. doi:10.2 9392/001c.12260

9. Ghana Statistical Service-GHS; ICF International. Ghana demographic health survey. Demographic and Health Survey 2014. Published online 2015. doi:10.151 71/ijhpm.2016.42
10. Brathwaite R, Addo J, Smeeth L, Lock K. A Systematic Review of Tobacco Smoking Prevalence and Description of Tobacco Control Strategies in SubSaharan African. PLoS ONE. Published online 2015.

11. Thompson E, Sofo S. Predicting Smoking Behaviors among Junior High School Students in Ghana. International Journal of Public Health Science (IJPHS). Published online 2018. doi:10.11591/ijphs.v7i $\underline{4.15225}$

12. Yawson AE, Baddoo A, Hagan-Seneadza NA, et al. Tobacco use in older adults in Ghana:

Sociodemographic characteristics, health risks and subjective wellbeing. BMC Public Health. Published online 2013. doi:10.1186/1471-2458-13-979

13. Dobbie F, Mdege N, Davidson F, et al. Building capacity for applied research to reduce tobaccorelated harm in low- and middle-income countries: the Tobacco Control Capacity Programme (TCCP). Journal of Global Health Reports. Published online 2019. doi:10.29392/joghr.3.e2019055

14. Eisenberg SL, Eisenberg MJ. Smoking Cessation During the COVID-19 Epidemic. Nicotine \& tobacco research: official journal of the Society for Research on Nicotine and Tobacco. Published online 2020. doi:10.1 $\underline{093 / n t r / n t a a 075}$

15. Farsalinos K, Niaura R, Le Houezec J, et al. Editorial: Nicotine and SARS-CoV-2: COVID-19 may be a disease of the nicotinic cholinergic system. Toxicology Reports. 2020;7:658-663.

16. Logo DD, Kyei-Faried S, Oppong FB, et al. Waterpipe use among the youth in Ghana: Lessons from the Global Youth Tobacco Survey (GYTS) 2017. Tobacco Induced Diseases. Published online 2020. do $\mathrm{i}: 10.18332 / \mathrm{TID} / 120937$

17. Rowell A. Coronavirus: big tobacco sees an opportunity in the pandemic. The Conversation. Published online 2020. https://theconversation.com/c oronavirus-big-tobacco-sees-an-opportunity-in-thepandemic- 138188 